\title{
NEUROPHYSIOLOGICAL CHARACTERIZATION OF PLANT- DERIVED DRUGS IN RATS AND HUMANS
}

\section{(c) Dimpfel W.}

Justus-Liebig-University Giessen, Germany c/o NeuroCode AG, 35578 Wetzlar, Sportparkstr. 9, Germany, Phone: 004964412002030, e-mail: w.dimpfel@neurocode-ag.com

Natural compounds and plant-derived products play an ever-increasing role not only in medical therapy but also in medical prophylaxis using food supplements and functional food. The topic of this presentation will try to bridge the gap between animal studies and clinical trials using an electrophysiological approach validated for years in testing pharmaceutical preparations of chemically synthesized compounds. Common parameter of analysis in rat and humans is the local field potential as it is recorded from the depth of the rat brain and from the scalp of the human brain. These potentials - when recorded in the presence of drugs - are called electropharmacograms. In the rat, field potentials are recorded from implanted steel electrodes and transmitted via a telemetric system wireless to the secondary amplifiers and further transmitted to evaluation units by means of a glass fibre. The experimental design consists in recording a pre-drug base line for $\mathbf{4 5}$ minutes before administration of the natural ingredients or plant extracts. Recording continues for the next 5 hours. Effects are described in \% change from pre-drug values with respect to 6 frequency ranges for each of the four brain areas: frontal cortex, hippocampus, striatum and reticular formation $=24$ variables. Extracts from decaffeinated green tea, Schisandra, Ginkgo, Ginseng, St. John's Wort, Radix Rhodiola, Valeriana, Camilla, Guarana, Passiflora, Kava-Kava were compared to the actions of caffeine, quercetin, theanine and theogallin. All extracts and single ingredients produced electrical changes which could be differentiated from each other by feed- ing the data into a discriminant analysis, where the first three discriminant axes were coded into spatial $x, y$ and $z$ axes. The next three discriminant axes were coded into an additive colour mixture of the colours green, red and blue. Using this method products with a similar effect on the brain provide a similar spatial location like seen for theanine, quercetin, St. John's Wort and give similar colours. In humans a similar approach is performed with data derived from EEG recordings. Development of a 16-channel EEG brain mapping system allowed online real time depiction of all frequency changes within one chart. Data from a lozenge containing four plant derived extracts (lemon balm, oats, lavender oil and hops), a drink containing ginkgo and ginseng as well as a decaffeinated green tea extract and a tablet containing passiflora were tested within a similar experimental design in comparison to placebo. After base line recording, administration of the extracts was followed by hourly recordings under conditions of eyes open as well as in the presence of different mental challenges. The data from the electropharmacograms (17 electrode positions and 6 frequency ranges $=102$ variables) were fed into discriminant analysis. It could be shown that the actions of the four preparations were entirely different from placebo. This proved the extremely high sensitivity of the method and the consistency of the observed effects. The data document that field potentials from rats and humans are valid parameters for the description of effects of natural compounds and herbal extracts on the brain.

\section{ANTIOXIDANT ACTIVITY AND PHENOLIC PROFILE OF SERBIAN HONEY WITH DRIED PLUMS}

\section{() Djilas Sonja, Čanadanović-Brunet Jasna, Ćetković Gordana, Tumbas Vesna, Vulić Jelena, Savatović Slađana}

Faculty of Technology, University of Novi Sad, Bulevar cara Lazara 1, 21000 Novi Sad, Serbia

Honey serves as a good source of natural antioxidants, which are effective in reducing the risk occurrence of heart disease, cancer, cataracts, different immunesystem decline, etc. Dried plums contain higher levels of polyphenols and show stronger antioxidant activity than most other fruits. The aim of this research was to investigate the contribution of addition (20-40\% mass) of dried plums to phenolic and flavonoid contents and radical scavenging activity (RSA) of lime honey - Serbian polyfloral honey with appellation of origin. Total phenolic
(TP) and flavonoid contents (TF) were determined spectrophotometricaly after 10 days after plums addition. Also, RSA on superoxide anion $\left(\mathrm{O}_{2}{ }^{*}\right)$ and hydroxyl radicals $(\cdot \mathrm{OH})$ were investigated by electron spin resonance spectroscopy. TP and TF in lime honey $(27.4 \mathrm{mg} / 100 \mathrm{~g}$ and $9.8 \mathrm{mg} / 100 \mathrm{~g}$, respectively) was increased after addition of dried plums in a dose dependent manner. Highest TP (61.4 mg/100g) and TF (38.5 mg/100g) were determined in honey sample with $40 \%$ of dried plums. RSA of lime honey $\left(\mathrm{EC}_{50} \mathrm{O}_{2}{ }^{*}=55.5 \mathrm{mg} / \mathrm{ml}\right.$; 